\title{
BMJ Open Metformin discontinuation in patients beginning second-line glucose-lowering therapy: results from the global observational DISCOVER study programme
}

Kamlesh Khunti (10 , ${ }^{1}$ Marilia B Gomes, ${ }^{2}$ Mikhail Kosiborod, ${ }^{3,4,5}$ Antonio Nicolucci, ${ }^{6}$ Stuart Pocock, ${ }^{7}$ Wolfgang Rathmann, ${ }^{8}$ Marina V Shestakova, ${ }^{9}$ lichiro Shimomura, ${ }^{10}$ Hirotaka Watada, ${ }^{11}$ Hungta Chen, ${ }^{12}$ Javier Cid-Ruzafa, ${ }^{13}$ Peter Fenici, ${ }^{14}$ Niklas Hammar, ${ }^{15,16}$ Fengming Tang, ${ }^{3}$ Linong $\mathrm{Ji}^{17}{ }^{17}$ On behalf of the DISCOVER Scientific Committee and all DISCOVER investigators

To cite: Khunti K, Gomes MB, Kosiborod M, et al. Metformin discontinuation in patients beginning second-line glucose-lowering therapy: results from the global observational DISCOVER study programme. BMJ Open 2020;10:e034613. doi:10.1136/ bmjopen-2019-034613

- Prepublication history and additional material for this paper are available online. To view these files, please visit the journal online (http://dx.doi. org/10.1136/bmjopen-2019034613).

Received 04 October 2019 Revised 28 April 2020 Accepted 20 May 2020
Check for updates

(C) Author(s) (or their employer(s)) 2020. Re-use permitted under CC BY-NC. No commercial re-use. See rights and permissions. Published by BMJ.

For numbered affiliations see end of article.

Correspondence to Professor Kamlesh Khunti; kk22@le.ac.uk

\section{ABSTRACT}

Objectives To evaluate the extent to which patients with type 2 diabetes discontinue metformin therapy when initiating second-line treatment and factors associated with metformin discontinuation, using baseline data from the DISCOVER study programme.

Design DISCOVER is a 3-year, prospective, observational study programme including data from 38 countries across 6 continents from 2014 to 2019.

Setting Primary and secondary healthcare centres, hospitals and specialist diabetes centres in both urban and rural locations.

Participants A total of 15992 patients with type 2 diabetes initiating second-line glucose-lowering therapy.

Primary and secondary outcome measures The proportion of patients who discontinued metformin as a second-line therapy and the factors associated with this treatment change.

Results Of the 14668 patients (from 37 countries) with valid treatment data, $11837(80.7 \%)$ received metformin as first-line glucose-lowering therapy; 8488 (71.7\%) received metformin monotherapy and $3349(28.3 \%)$ received metformin as part of a combination therapy. Overall, treatment with metformin was discontinued in $15.1 \%$ (1782) of patients who received first-line metformin (14.1\% (1194) and $17.6 \%(588)$ in those who received metformin as monotherapy and as part of a combination, respectively); this proportion varied across regions from $6.9 \%$ (54) in Africa to $20.6 \%$ (628) in South-East Asia. On metformin discontinuation, $73.6 \%$ (1311) of patients received a non-insulin monotherapy at second line. Factors associated with an increased odds of metformin discontinuation were older age ( $\geq 75$ years) and having a history of chronic kidney disease. The probability of metformin monotherapy discontinuation was lower in patients from Africa than in those from Europe.

Conclusions A substantial number of patients discontinued taking metformin when beginning secondline therapy. Most of these patients subsequently received a non-insulin monotherapy at second line, in contradiction

\section{Strengths and limitations of this study}

- The study involved countries from which limited data are available on type 2 diabetes.

- The standardised case report form allowed comparisons to be made between regions.

- The diversity of sites provides a picture of global healthcare settings.

- Quality of care in some countries may still be overrepresented or under-represented.

- Not all patients had data collected for all study variables.

to international guidelines and potentially leaving them at an increased risk of hyperglycaemia and associated adverse outcomes.

Trial registration numbers NCT02322762 and NCT02226822.

\section{INTRODUCTION}

Current international clinical guidelines for the treatment of patients with type 2 diabetes recommend the use of metformin for most patients as the first-line pharmacological treatment in conjunction with lifestyle changes to control glycaemic levels. ${ }^{1-6}$ If glycated haemoglobin $\left(\mathrm{HbA}_{1 \mathrm{c}}\right)$ levels remain uncontrolled, guidelines recommend the addition of a second glucose-lowering agent to ongoing metformin therapy. The proportion of patients who discontinue metformin treatment, and reasons for discontinuation, have not previously been assessed on a global scale.

DISCOVER is a 3-year, global, prospective, non-interventional study programme conducted simultaneously in 38 countries 
across 6 continents, recruiting patients with type 2 diabetes initiating a second-line glucose-lowering therapy (defined as adding a glucose-lowering drug or switching between therapies). Comprehensive data were collected using a standardised electronic case report form. The primary study objectives are to describe disease management patterns and associated clinical and health-related quality of life outcomes. ${ }^{7}$ In this study we aimed to evaluate the extent to which patients discontinue treatment with metformin when initiating a second-line glucose-lowering therapy. Factors associated with metformin discontinuation were also assessed.

\section{METHODS}

The methods for the DISCOVER study programme have been described in detail elsewhere ${ }^{7}$ and are briefly summarised below.

\section{Study design}

The DISCOVER study programme comprises two similar, 3-year, non-interventional, prospective studies conducted in parallel in 38 countries and including a total of 15992 patients: DISCOVER in 37 countries and J-DISCOVER in Japan. Countries are grouped into regions according to WHO categories: ${ }^{8}$ Africa (Algeria and South Africa); Americas (Argentina, Brazil, Canada, Colombia, Costa Rica, Mexico and Panama); South-East Asia (India and Indonesia); Europe (Austria, Czech Republic, Denmark, France, Italy, Netherlands, Norway, Poland, Russia, Spain, Sweden and Turkey); the Eastern Mediterranean (Bahrain, Egypt, Jordan, Kuwait, Lebanon, Oman, Saudi Arabia, Tunisia and United Arab Emirates); and the Western Pacific (Australia, China, Japan, Malaysia, South Korea and Taiwan).

The study protocols were approved by the relevant clinical research ethics committees in each country and institutional review boards at each site (online supplementary appendix), and complied with the Declaration of Helsinki, the International Conference on Harmonisation Guideline for Good Clinical Practice, and the local regulations for clinical research.

\section{Patient and public involvement}

Patients or the public were not involved in the design, or conduct, or reporting or dissemination of our research.

\section{Site and investigator selection}

For each participating country, characteristics of the physicians and clinical centres involved in the management of patients with type 2 diabetes were first assessed by conducting literature searches and interviewing key local diabetes experts, who acted as the national coordinating investigators for the study. Information on the proportions of physicians by speciality (primary care practitioners, diabetologists/endocrinologists, cardiologists and other specialities) and types of practices (primary care centres, specialised diabetes centres and different types of hospitals) in each country was collated. A list of sites that would match these characteristics as closely as possible was established for each country, and the sites were invited to participate. Approximately one-third of invited sites were able to participate and enrolled patients in the study.

\section{Patient enrolment}

Inclusion and exclusion criteria were kept to a minimum to reflect the diversity of patients treated in routine clinical practice. Briefly, patients aged 18 years or older who had type 2 diabetes and who were initiating a secondline glucose-lowering therapy were eligible for inclusion if they were not pregnant, were not undergoing dialysis and did not have a history of renal transplant, and if their first-line therapy was not an injectable agent or a herbal remedy or natural medicine alone. Full inclusion and exclusion criteria can be found in online supplementary table S1. The study protocol stated that investigating physicians should invite consecutive eligible patients to enrol in the study. All participating patients provided written informed consent.

\section{Data collection}

Data were collected using a standardised electronic case report form and transferred to a central database via a webbased data capture system. Some data were extracted from existing electronic health records in Canada, Denmark, France, Norway and Sweden. In these countries, data not routinely captured in electronic medical records, such as the reason(s) for treatment change, were obtained by the investigators using a questionnaire that was linked back to patients' medical records. Variables collected at baseline included physician and site characteristics, patient demographics, physiological parameters, laboratory test results including $\mathrm{HbA}_{1 \mathrm{c}}$ level, change in glucose-lowering therapies and reason(s) for change, comorbidities including microvascular and macrovascular diseases and co-medications. In line with the observational nature of the study, clinical variables such as $\mathrm{HbA}_{1 \mathrm{c}}$ and/or fasting plasma glucose levels were measured and recorded in accordance with routine clinical practice; data collection was not mandatory for any of the clinical variables.

\section{Statistical analysis}

For the present analysis patients from China $(n=1293)$ were excluded because complete data were not available at the time of publication. Patients were also excluded from the analysis if they had treatment information missing at baseline $(n=4)$ or if they had metformin monotherapy recorded as both first-line and secondline therapy $(n=27)$. Data from a total of 14668 patients from DISCOVER were therefore assessed for this analysis. Descriptive data are reported as numbers and percentages, with percentages calculated using the total number of patients with data available as the denominator. Mean (SD) values and across-region ranges (ARRs) are also reported when appropriate. Factors associated with 
metformin discontinuation were assessed using hierarchical logistical models with country as a random effect and with baseline covariates (sex, age, body mass index, education level, time since diagnosis, $\mathrm{HbA}_{1 \mathrm{c}}$ level, health insurance status, employment status, region and medical history: macrovascular complications, microvascular complications, chronic kidney disease (CKD) and minor or major hypoglycaemic events) as fixed effects. These covariates were selected a priori as clinically relevant variables that could potentially be associated with the odds of metformin discontinuation.

Median ORs (MORs) were calculated to describe country-level variance in the odds of metformin discontinuation. The MOR is the median of the ORs obtained when comparing the odds of discontinuing metformin in an individual from a randomly selected country with that of another individual with identical baseline covariates from another randomly selected country. The MOR was calculated by assessing the odds of metformin discontinuation for all the selected pairs of individuals from different countries, with the individual from the country with the highest odds of metformin discontinuation as the numerator and the individual with the lowest odds of metformin discontinuation as the denominator. This calculation resulted in a distribution of ORs always $\geq 1$. The MOR is the median value of this distribution. ${ }^{9}{ }^{10}$ If the MOR was equal to 1 , there would be no difference between countries in the probability of discontinuing metformin; the larger the MOR, the greater the variability in the probabilities of discontinuing metformin across countries. All statistical analyses were conducted using the SAS 9.4 statistical software system (SAS Institute Inc, Cary, North Carolina, USA).

\section{RESULTS}

\section{Patient baseline characteristics}

Of the 14668 DISCOVER participants with valid treatment data, 11837 (80.7\%) had received metformin (on its own or as part of a combination) as first-line treatment (table 1). Some variation in baseline characteristics of patients receiving metformin as a first-line therapy was seen across study regions (online supplementary file 2).

Among the patients who received first-line metformin, 10055 (84.9\%, ARR: $79.4 \%$ to $93.1 \%)$ continued metformin at second line, while 1782 (15.1\%, ARR: $6.9 \%$ to $20.6 \%$ ) discontinued metformin. Most baseline characteristics were similar between patients who continued and those who discontinued treatment. The proportion of patients with a history of microvascular complications was lower in patients continuing metformin $(17.5 \%)$ than in those discontinuing metformin $(21.6 \%)$. The proportion of patients with a history of CKD was also lower in patients continuing metformin $(3.3 \%)$ than in those discontinuing metformin $(6.6 \%)$. The proportion of patients with health insurance was higher in patients continuing metformin $(75.9 \%)$ than in those discontinuing metformin $(66.2 \%)$.

\section{Treatment patterns}

Second-line treatment patterns for the 11837 patients who had received metformin at first line are shown according to first-line treatment in table 2 , and according to region in table 3. Of these patients, 8488 (71.7\%) had received first-line metformin monotherapy. The remaining 3349 patients $(28.3 \%)$ had received metformin as part of a combination therapy, most commonly with a sulphonylurea $(63.9 \%)$. First-line metformin was most commonly prescribed as a monotherapy in all study regions other than South-East Asia (table 3).

The proportion of patients who discontinued metformin varied substantially across study regions, from $6.9 \%$ in Africa to $20.6 \%$ in South-East Asia (table 3). Among the 1782 patients (15.1\%) who discontinued treatment with metformin (monotherapy or as part of a combination therapy), the most common second-line therapies were dipeptidyl peptidase- 4 inhibitor monotherapies and sulphonylurea monotherapies $(27.0 \%$ and $20.3 \%$, respectively).

\section{Reasons for changing therapy}

Reasons for changing from first-line to second-line therapy (with or without metformin) are shown overall in table 4 and by region in online supplementary table S3. Lack of efficacy was the reason stated for changing therapy among $88.7 \%$ of patients (10 185/11 837) overall; $91.5 \%$ of patients (8928/10 055) who continued metformin therapy and $72.7 \%$ of patients $(1257 / 1782)$ who discontinued metformin therapy. Reasons for changing therapy also varied greatly across regions (online supplementary table S3). The proportions of patients who discontinued metformin for whom lack of efficacy was reported as a reason for treatment change ranged between $50.9 \%$ in the Americas and $94.4 \%$ in Africa.

Adverse effects were more commonly cited as a reason for changing therapy in patients who discontinued metformin $(16.8 \%)$ than in those who continued metformin (3.4\%). Chronic kidney disease development was also more commonly cited as a reason for changing therapy in patients who discontinued metformin $(4.7 \%)$ than in those who continued metformin $(0.3 \%)$. Of the patients who discontinued metformin, the proportions of patients for whom adverse effects were cited as reasons for changing therapy ranged from $1.9 \%$ in Africa to $41.4 \%$ in the Americas; the proportions of patients for whom chronic disease development was given as a reason for changing therapy ranged from $0.0 \%$ in Africa to $10.8 \%$ in Europe.

\section{Factors associated with metformin therapy discontinuation}

Factors associated with increased or decreased odds of discontinuing metformin are shown in figure 1. Having CKD and being aged 75 years or older (vs $<65$ years) were associated with an increased odds of metformin discontinuation, whether metformin was used as a monotherapy or as part of a combination. In addition, among patients who had received first-line metformin monotherapy, the 
Table 1 Baseline characteristics of patients receiving a second-line therapy with or without metformin

\begin{tabular}{|c|c|c|c|c|}
\hline Characteristic & $\begin{array}{l}\text { Second-line therapy with } \\
\text { MET } n=10055\end{array}$ & $\begin{array}{l}\text { Second-line therapy without } \\
\text { MET } n=1782\end{array}$ & Overall $\mathrm{N}=11837$ & $P$ value \\
\hline Proportion of patients, \% & 84.9 & 15.1 & 100 & \\
\hline Sex, male, n (\%) & $5321(52.9)$ & $953(53.5)$ & $6274(53.0)$ & 0.574 \\
\hline Missing, $\mathrm{n}$ & 2 & 2 & 4 & \\
\hline \multicolumn{5}{|l|}{ Age } \\
\hline Years, mean (SD) & $56.2(11.6)$ & $57.8(12.1)$ & $56.5(11.7)$ & 0.179 \\
\hline 66-75 years, n (\%) & $1704(16.9)$ & $327(18.4)$ & $2031(17.2)$ & \\
\hline$\geq 76$ years, $n(\%)$ & $416(4.1)$ & $122(6.8)$ & $538(4.5)$ & \\
\hline Missing, $n$ & 0 & 0 & 0 & \\
\hline BMI, kg/m², mean (SD) & $30.0(5.9)$ & $29.7(6.3)$ & $30.0(6.0)$ & $<0.001$ \\
\hline Missing, $\mathrm{n}$ & 863 & 140 & 1003 & \\
\hline 7.0 to $<8.0, \mathrm{n}(\%)$ & $2559(31.6)$ & $392(29.9)$ & $2951(31.3)$ & \\
\hline 8.0 to $<9.0, \mathrm{n}(\%)$ & $2012(24.8)$ & $272(20.7)$ & $2284(24.3)$ & \\
\hline$\geq 9.0, \mathrm{n}(\%)$ & $2404(29.7)$ & $314(23.9)$ & $2718(28.9)$ & \\
\hline Missing, $n$ & 1950 & 469 & 2419 & \\
\hline \multicolumn{5}{|l|}{ Education level, n (\%) } \\
\hline No formal education & $290(3.2)$ & $44(2.7)$ & $334(3.1)$ & $<0.001$ \\
\hline Primary education (1-6 years) & $1599(17.5)$ & $228(14.0)$ & $1827(17.0)$ & \\
\hline Secondary education ( $7-13$ years) & $4262(46.7)$ & $847(52.0)$ & $5109(47.5)$ & \\
\hline$>13$ years of education & $2971(32.6)$ & $510(31.3)$ & $3481(32.4)$ & \\
\hline Employed or self-employed, $n$ (\%) & $4636(49.2)$ & $771(45.9)$ & $5407(48.7)$ & 0.573 \\
\hline Missing & 639 & 102 & 741 & \\
\hline \multicolumn{5}{|l|}{ Medical history, $\mathrm{n}(\%)$} \\
\hline Macrovascular complications & $1380(13.8)$ & $259(14.6)$ & $1639(13.9)$ & 0.001 \\
\hline Missing, $\mathrm{n}$ & 31 & 7 & 38 & \\
\hline Microvascular complications & $1755(17.5)$ & $384(21.6)$ & $2139(18.1)$ & 0.010 \\
\hline Missing, $\mathrm{n}$ & 12 & 3 & 15 & \\
\hline Chronic kidney disease, $\mathrm{n}(\%)$ & $329(3.3)$ & $117(6.6)$ & $446(3.8)$ & 0.831 \\
\hline Stage 2 (eGFR $60-89 \mathrm{~mL} / \mathrm{min} / 1.73 \mathrm{~m}^{2}$ ) & $181(55.0)$ & $52(44.4)$ & $233(52.2)$ & $<0.001$ \\
\hline Stage 3 (eGFR $30-59 \mathrm{~mL} / \mathrm{min} / 1.73 \mathrm{~m}^{2}$ ) & $91(27.7)$ & $50(42.7)$ & $141(31.6)$ & \\
\hline Stage 4 (eGFR $\left.15-29 \mathrm{~mL} / \mathrm{min} / 1.73 \mathrm{~m}^{2}\right)$ & $6(1.8)$ & $8(6.8)$ & $14(3.1)$ & \\
\hline Stage 5 (eGFR $<15 \mathrm{~mL} / \mathrm{min} / 1.73 \mathrm{~m}^{2}$ ) & $6(1.8)$ & $0(0.0)$ & $6(1.3)$ & \\
\hline Stage unknown & $45(13.7)$ & $7(6.0)$ & $52(11.7)$ & \\
\hline Missing, $\mathrm{n}$ & 12 & 3 & 15 & \\
\hline Time since diagnosis, years, mean (SD) & $5.6(5.1)$ & $5.3(4.8)$ & $5.6(5.1)$ & $<0.001$ \\
\hline Missing, $\mathrm{n}$ & 200 & 42 & 242 & \\
\hline
\end{tabular}

Data are reported as $\mathrm{n}(\%)$, unless otherwise stated. Percentages were calculated for all patients with data available; patients with missing data were excluded.

$P$ values were calculated using either a Student's t-test (continuous variables), $\chi^{2}$ test (categorical variables) or Fisher's exact test (categorical variables). $P$ values $<0.05$ were considered statistically significant.

BMI, body mass index; eGFR, estimated glomerular filtration rate; $\mathrm{HbA}_{1 c}$, glycated haemoglobin; MET, metformin. 
Table 2 Second-line therapies in patients receiving metformin (as monotherapy or as part of a combination treatment) as a first-line therapy, according to first-line therapy

\begin{tabular}{|c|c|c|c|c|c|c|}
\hline \multirow[b]{2}{*}{ Second-line therapy } & \multicolumn{5}{|c|}{ First-line therapy } & \multirow[b]{2}{*}{$\begin{array}{l}\text { Overall } \\
n=11837\end{array}$} \\
\hline & $\begin{array}{l}\text { MET } \\
\text { monotherapy } \\
\mathrm{n}=8488 \text { (71.7) }\end{array}$ & $\begin{array}{l}\text { MET+SU } \\
n=2139 \\
(18.1)\end{array}$ & $\begin{array}{l}\text { MET+DPP-4i } \\
\mathrm{n}=489(4.1)\end{array}$ & $\begin{array}{l}\text { MET+SU+ } \\
\text { DDP-4i n=217 } \\
(1.8)\end{array}$ & $\begin{array}{l}\text { MET+other(s) } \\
\mathrm{n}=504(4.3)\end{array}$ & \\
\hline Second-line therapy with MET & 7294 (85.9) & 1739 (81.3) & $406(83.0)$ & $184(84.8)$ & $432(85.7)$ & $10055(84.9)$ \\
\hline MET monotherapy & $0(0.0)$ & $22(1.0)$ & $9(1.8)$ & $2(0.9)$ & $2(0.4)$ & $35(0.3)$ \\
\hline $\mathrm{MET}+\mathrm{SU}$ & $2549(30.0)$ & $137(6.4)$ & $23(4.7)$ & $2(0.9)$ & $31(6.2)$ & $2742(23.2)$ \\
\hline$M E T+S U+D P P-4 i$ & $135(1.6)$ & $588(27.5)$ & $131(26.8)$ & $29(13.4)$ & 37 (7.3) & $920(7.8)$ \\
\hline MET+insulin* & $288(3.4)$ & $214(10.0)$ & $33(6.7)$ & $59(27.2)$ & $79(15.7)$ & $673(5.7)$ \\
\hline MET+other(s) $†$ & $1542(18.2)$ & $535(25.0)$ & $152(31.1)$ & $89(41.0)$ & $227(45.0)$ & $2545(21.5)$ \\
\hline $\begin{array}{l}\text { Second-line therapy without } \\
\text { MET }\end{array}$ & $1194(14.1)$ & $400(18.7)$ & $83(17.0)$ & $33(15.2)$ & $72(14.3)$ & $1782(15.1)$ \\
\hline Insulin* & $61(0.7)$ & $52(2.4)$ & $13(2.7)$ & $9(4.1)$ & $13(2.6)$ & $148(1.3)$ \\
\hline $\begin{array}{l}\text { Other combinations without } \\
\text { MET }\end{array}$ & $211(2.5)$ & $59(2.8)$ & $22(4.5)$ & $9(4.1)$ & $22(4.4)$ & $323(2.7)$ \\
\hline
\end{tabular}

Data are reported as $\mathrm{n}(\%)$. Percentages were calculated for all patients with data available; patients with missing data were excluded. Switches between two agents in the same class (eg, from an SU to another SU) were considered as treatment changes.

*May contain other oral treatments.

†Including $\alpha$-glucosidase inhibitors, thiazolidinediones, meglitinides, sodium-glucose co-transporter-2 inhibitors and glucagon-like peptide-1 receptor agonists.

DPP-4i, dipeptidyl peptidase-4 inhibitor; MET, metformin; SU, sulphonylurea.

odds of metformin discontinuation were increased in patients who had experienced a major hypoglycaemic event or had a history of microvascular complications. The odds of metformin discontinuation were decreased in patients with a $\mathrm{HbA}_{1 \mathrm{c}}$ level $\geq 7.0 \%(53 \mathrm{mmol} / \mathrm{mol})$ who were prescribed first-line metformin monotherapy. The odds of discontinuing metformin monotherapy were also lower in patients from Africa than in those from Europe. Among patients who received first-line metformin as a combination therapy, the odds of metformin discontinuation were higher in patients who did not have health insurance than in those who did. No significant differences were observed across regions in the odds of discontinuing metformin as a combination therapy. The odds of metformin discontinuation varied substantially between countries as described by the MOR, used to assess the association between a patient's country of residence and the discontinuation of metformin treatment. Country was shown to be associated with metformin discontinuation in patients prescribed first-line metformin as a monotherapy (MOR: 2.55) and as part of a combination therapy (MOR: 2.58).

\section{DISCUSSION}

DISCOVER is a global, prospective study programme that assesses disease management, treatment patterns and clinical outcomes in patients with type 2 diabetes initiating second-line glucose-lowering therapy. This analysis of baseline data from DISCOVER showed that, of the patients who had received metformin as a firstline therapy, $84.9 \%$ continued with metformin as part of the second-line treatment. Nevertheless, a substantial proportion of patients $(15.1 \%)$ received secondline therapy without metformin, a higher percentage than would be expected if adhering to current clinical guidelines. ${ }^{3}$

Surprisingly, $14.1 \%$ of patients who received firstline metformin monotherapy discontinued metformin, despite guidelines recommending add-on glucoselowering drugs when metformin monotherapy fails to control glycaemia. ${ }^{3611}$ However, it is important to note that lack of efficacy is not the only reason for changing from first-line to second-line therapy. Previous studies have reported nausea, diarrhoea, stomach discomfort and a metallic taste in the mouth as potential adverse effects of metformin treatment, ${ }^{12}$ which may lead to metformin discontinuation. In our analyses, adverse effects were stated as a reason for changing from first-line therapy in a higher proportion of patients who discontinued $(16.8 \%)$ than continued metformin $(3.4 \%)$. This may partly explain the large proportion of patients who had discontinued metformin monotherapy. 
Table 3 First-line and second-line therapies in patients receiving metformin (as monotherapy or as part of a combination treatment) as a first-line therapy, according to study region

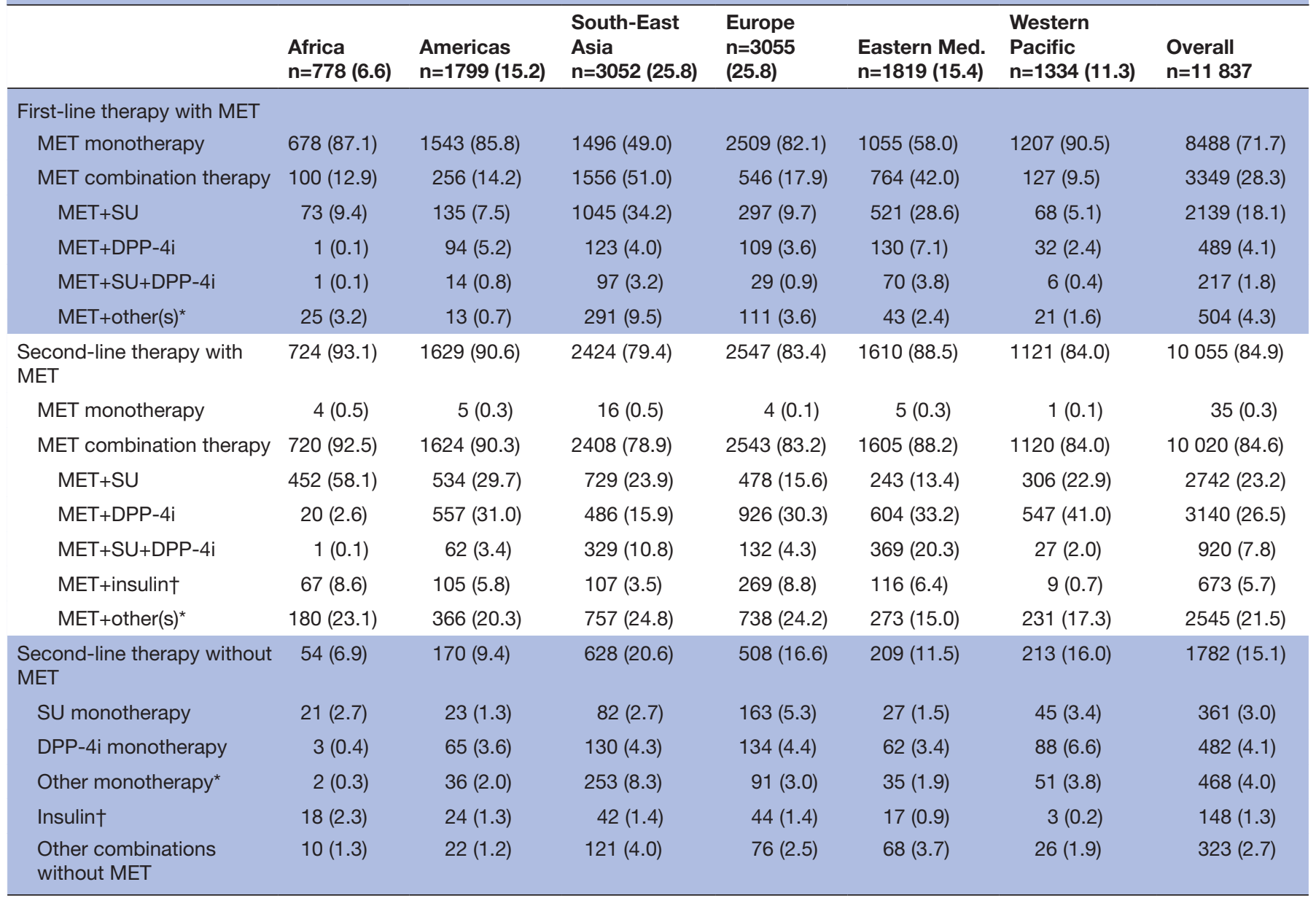

Data are reported as $n(\%)$. Percentages were calculated for all patients with data available; patients with missing data were excluded. Countries were grouped according to the WHO regional classification (Africa: Algeria and South Africa; Americas: Argentina, Brazil, Canada, Colombia, Costa Rica, Mexico and Panama; South-East Asia: India and Indonesia; Europe: Austria, Czech Republic, Denmark, France, Italy, Netherlands, Norway, Poland, Russia, Spain, Sweden and Turkey; Eastern Mediterranean: Bahrain, Egypt, Jordan, Kuwait, Lebanon, Oman, Saudi Arabia, Tunisia and United Arab Emirates; Western Pacific: Australia, Japan, Malaysia, South Korea and Taiwan).

*Including $\alpha$-glucosidase inhibitors, thiazolidinediones, meglitinides, sodium-glucose co-transporter-2 inhibitors and glucagon-like peptide-1 receptor agonists.

†May contain other oral treatments.

DPP-4i, dipeptidyl peptidase-4 inhibitor; Med, Mediterranean; MET, metformin; SU, sulphonylurea.

Factors associated with the discontinuation of metformin as a second-line therapy varied between patients prescribed first-line metformin as a monotherapy and those receiving it as a combination therapy. Among patients who had received first-line metformin as part of a combination therapy, the odds of metformin discontinuation were higher in those with no health insurance than in those who had health insurance. Because the cost of metformin is relatively low compared with other glucoselowering therapies, this is unlikely to be a key reason for treatment discontinuation in uninsured patients. Patients with a history of CKD were also more likely to discontinue the metformin therapy than those without CKD. In accordance with this, the prevalence of CKD in our study population at baseline was higher in patients initiating a second-line therapy without metformin than in those continuing treatment with metformin. This is in line with current guidelines that recommend against prescribing metformin to patients who have an estimated glomerular filtration rate (eGFR) lower than $30 \mathrm{~mL} / \mathrm{min} / 1.73 \mathrm{~m}^{2}$ (ie, stage 4 or stage $5 \mathrm{CKD}$ ), and recommend decreasing the dose for patients with mild-to-moderate renal impairment (eGFR 30 to $59 \mathrm{~mL} / \mathrm{min} / 1.73 \mathrm{~m}^{2}$, stage 3 CKD).${ }^{3613-15}$ However, in a recent phase 1 trial in patients with type 2 diabetes and stage $4 \mathrm{CKD}$, treatment with lowdose metformin was not associated with adverse safety outcomes, ${ }^{16}$ suggesting that metformin discontinuation may not be necessary for these patients.

The proportions of patients who discontinued metformin varied considerably across study regions, from $6.9 \%$ in Africa to $20.6 \%$ in South-East Asia. Despite this variation, region was not associated with a significantly increased chance of discontinuing metformin in the multivariable analysis other than in Africa, where patients 
Table 4 Reasons cited by physicians for changing therapy in patients receiving metformin (as monotherapy or as part of a combination treatment) as a first-line therapy, for patients receiving a second-line therapy with or without metformin

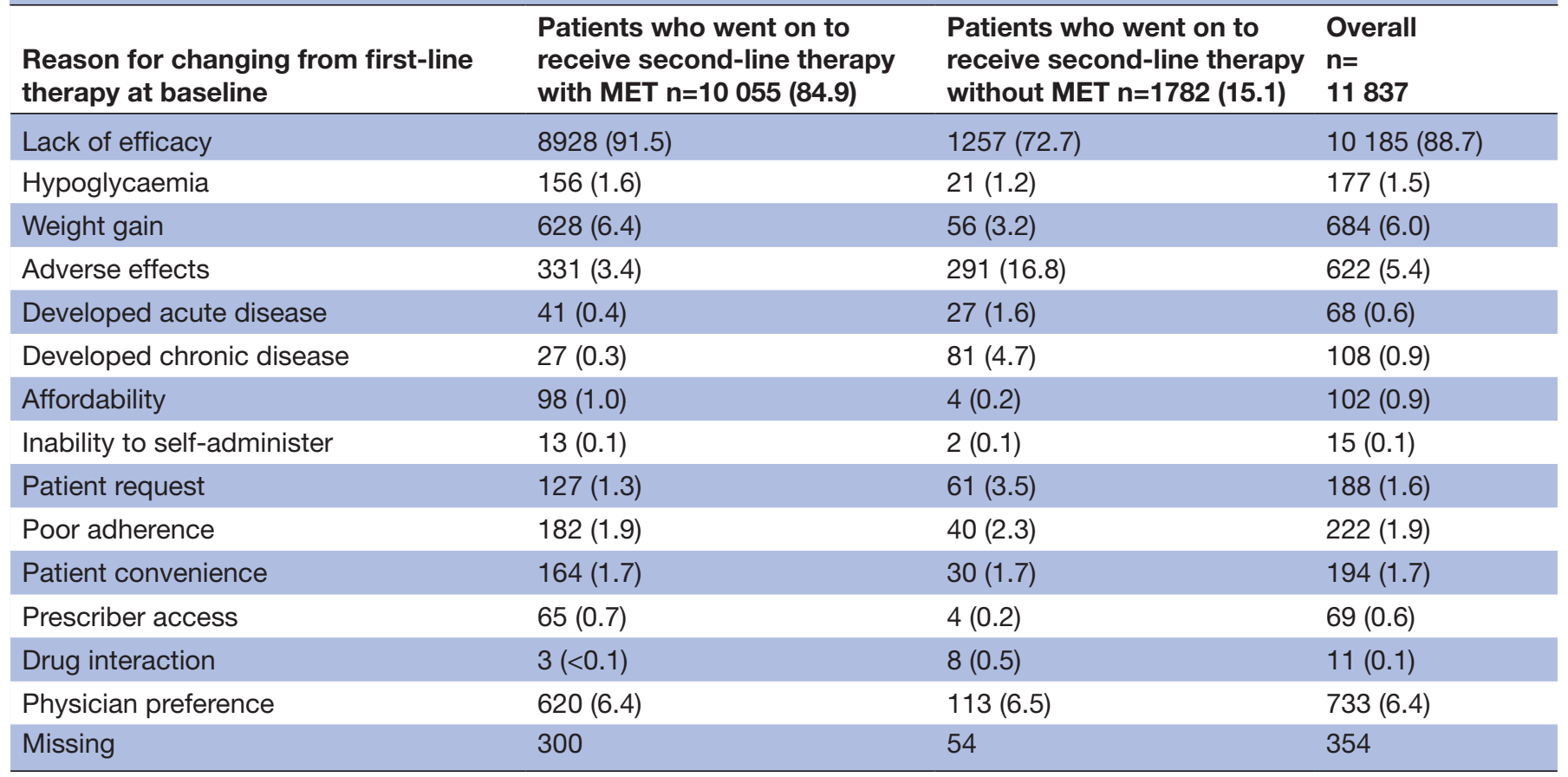

Data are reported as $\mathrm{n}(\%)$. Several reasons could be selected for each patient. Percentages were calculated for all patients with data available; patients with missing data were excluded. Overall, data were available for 11483 patients (9755 and 1728 patients who received second-line therapy with and without MET, respectively, after exclusion of missing data). MET, metformin.

who had received first-line metformin monotherapy were less likely to discontinue metformin than patients from Europe. However, as shown by the MOR, countrylevel variation was associated with a higher chance of discontinuing metformin, suggesting that the differences in the proportions of patients prescribed a second-line treatment without metformin are mainly due to countrylevel rather than regional variation. A previous study of treatment patterns across five European countries also found substantial variation in second-line treatment choice between countries, possibly reflecting differences in local treatment guidelines. ${ }^{17}$ Country-level variation may also be explained by differences in the availability and affordability of different glucose-lowering drugs. A study published in 2018 found that metformin availability ranged between $100 \%$ in high-income countries to $64.7 \%$ in low-income countries. ${ }^{18}$ However, the availability of metformin in low-income countries was still higher than that of other glucose-lowering drugs such as insulin, available to only $10.3 \%$ of patients. The study also estimated that while $26.9 \%$ of patients from low-income countries could not afford metformin, $63.0 \%$ were unable to afford insulin, showing an association between both affordability and accessibility and the use of diabetes medications.

To our knowledge, the effect of metformin discontinuation on patient outcomes when initiating a second-line glucose-lowering therapy have not been extensively studied. A retrospective study of electronic medical records in the USA showed that metformin discontinuation in patients with declining renal function was associated with weight gain and increased risk of hypoglycaemia. ${ }^{19}$ This is likely explained by the fact that most of these patients switched from metformin monotherapy to a sulphonylurea or insulin; ${ }^{19}$ these switches were observed in approximately one-third of DISCOVER patients who were using metformin monotherapy, regardless of their renal function. Future analyses of DISCOVER follow-up data may provide useful insights into the association between metformin discontinuation and clinical outcomes.

\section{Strengths and limitations}

The key strengths of the DISCOVER study programme include the large number of participating countries, sites and patients, as well as the inclusion of many countries for which little or no data on the treatment of patients with type 2 diabetes are currently available. Data were collected using a standardised case report form, thereby allowing direct comparison between countries and regions. In addition, the wide diversity of sites and specialities of investigators helps to provide as inclusive a view as possible of the variety of healthcare settings across the world.

As previously reported, some limitations of the DISCOVER study should be considered. ${ }^{7}$ In accordance with the observational nature of the study, the protocol did not mandate the collection and recording of study variables, resulting in missing data for some variables 


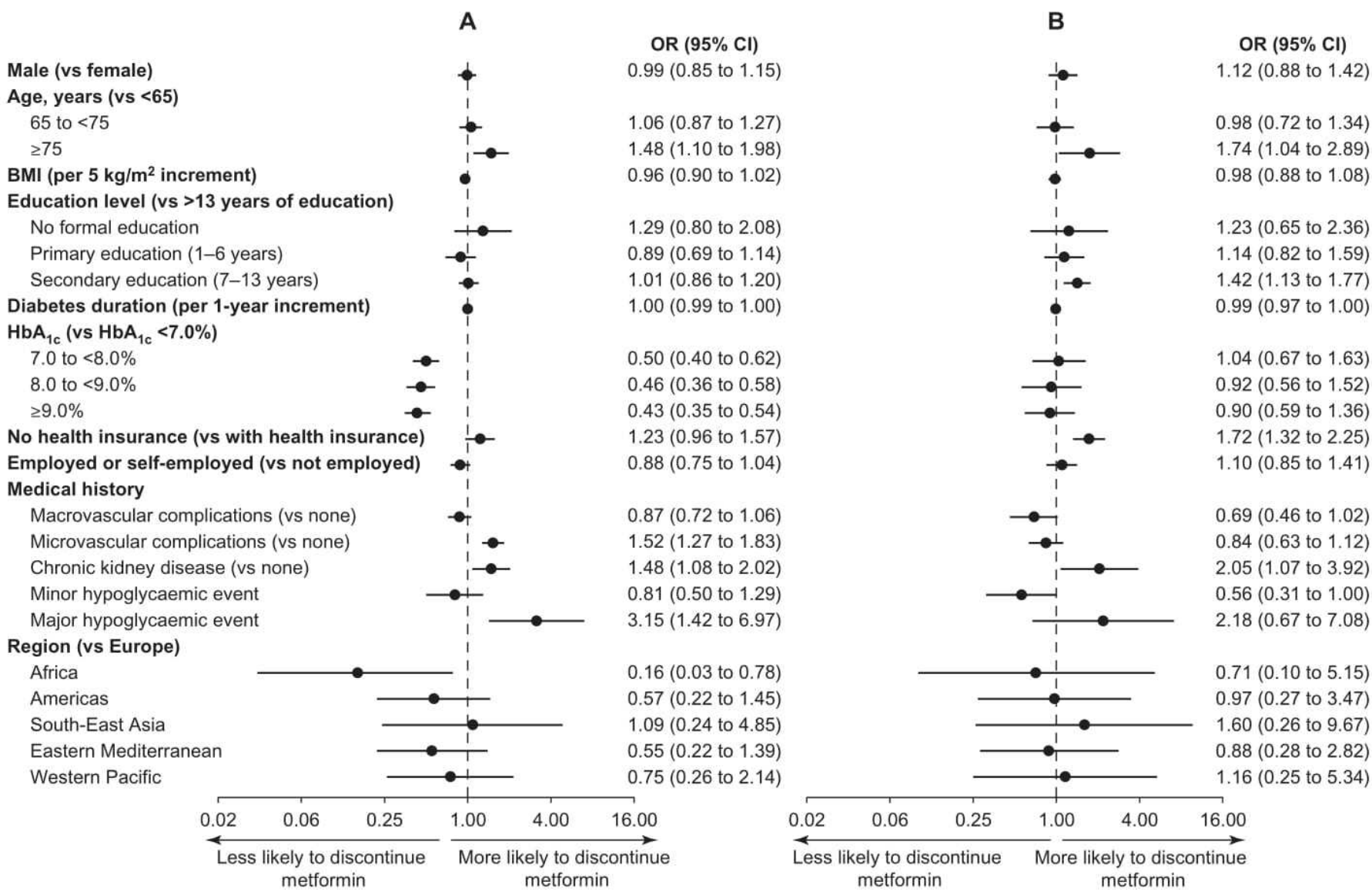

Figure 1 Variables associated with metformin discontinuation in patients receiving metformin (A) as monotherapy and (B) as part of a combination as first-line therapy. ORs were calculated using a hierarchical logistic regression model, with country as a random effect and adjusted for all variables in the figure. $\mathrm{BMI}$, body mass index; $\mathrm{HbA}_{10}$, glycated haemoglobin.

for some patients. However, missing data for variables such as $\mathrm{HbA}_{1 \mathrm{c}}$ and cholesterol levels reflect real-world clinical practice and thereby enable DISCOVER data to be used to examine how differences in clinical practice across and within countries affect disease management and outcomes. As for any observational study, our findings should also be interpreted in the context of potential residual confounding. Although the multivariate analyses were adjusted for a number of covariates, other variables for which data were not collected (eg, detailed information about insurance coverage, access to treatments and study site funding) could also be associated with the odds of metformin discontinuation.

Finally, DISCOVER sites were selected to optimise diversity in each country, but it was not always possible to achieve full representativeness of the general patient population at a country, regional and global level. Reasons for this include a lack of comprehensive country-level data on the types of sites and physicians treating patients with type 2 diabetes before the start of the study, the small total number of sites in some countries and reduced participation of rural and primary care centres because of infrastructural and site capability limitations. ${ }^{20}$ The result may be an over-representation of more advanced treatment in the urban and secondary care centres, potentially leading to an overestimation of the quality of care in some countries as well as to an over-representation of patients with more advanced disease and comorbidities.

\section{Conclusion}

In conclusion, a substantial proportion of DISCOVER patients who were prescribed first-line metformin discontinued therapy; the proportions of patients who discontinued metformin therapy differed between study regions. Most of these patients subsequently received a non-insulin monotherapy at second line, in contradiction to international guidelines and potentially leaving them at an increased risk of hyperglycaemia and associated adverse outcomes. Differences in second-line treatment choice between countries could be explained by local treatment availability and/or affordability, or by differences in local guidelines and practices. Further analyses from the DISCOVER study will build on the data presented here, to provide a comprehensive picture of treatment patterns and outcomes from second-line to later-line therapies across a variety of healthcare settings.

\section{Author affiliations}

${ }^{1}$ Diabetes Research Centre, University of Leicester, Leicester, UK

${ }^{2}$ Department of Internal Medicine, Rio de Janeiro State University, Rio de Janeiro, RJ, Brazil

${ }^{3}$ Department of Cardiovascular research, Saint Luke's Mid America Heart Institute, Kansas City, Missouri, USA 
${ }^{4}$ Department of Internal Medicine, University of Missouri Kansas City, Kansas City, Missouri, USA

${ }^{5}$ The George Institute for Global Health, University of New South Wales, Sydney, New South Wales, Australia

${ }^{6}$ Department of Metabolic Medicine, Center for Outcomes Research and clinical Epidemiology (CORE), Pescara, Italy

${ }^{7}$ Department of Medical Statistics, London School of Hygiene and Tropical Medicine, London, UK

${ }^{8}$ Institute for Biometrics and Epidemiology, German Diabetes Center, Düsseldorf, Germany

${ }^{9}$ Feberal Scientific Centre of Endocrinology, Endocrinology Research Centre, Moscow, Russian Federation

${ }^{10}$ Department of Metabolic Medicine, Osaka University Hospital, Suita, Japan

${ }^{11}$ Department of Metabolism and Endocrinology, Juntendo University, Bunkyo-ku,

Tokyo, Japan

${ }^{12}$ AstraZeneca, Gaithersburg, Maryland, USA

${ }^{13}$ Evidera, Barcelona, Spain

${ }^{14}$ AstraZeneca, Cambridge, UK

${ }^{15}$ Institute of Environmental Medicine, Karolinska Institutet, Stockholm, Sweden

${ }^{16}$ AstraZeneca, Gothenburg, Sweden

${ }^{17}$ Department of Endocrinology and Metabolism, Peking University People's Hospital, Beijing, China

Acknowledgements The authors would like to thank all the patients and investigators participating in the DISCOVER study programme. Medical writing support was provided by Jessica Horsburgh, PhD, and Steph Macdonald, PhD, of Oxford PharmaGenesis, Oxford, UK, and was funded by AstraZeneca.

Contributors KK, MBG, MK, AN, SP, WR, MVS, IS, HW, HC, JCR, PF, NH, FT and $\mathrm{LJ}$ agreed the general content of the manuscript. KK, HC and FT developed the statistical analysis plan, which was conducted by $\mathrm{HC}$ and FT. The first draft of the manuscript was developed by KK, with input from MBG, MK, AN, SP, WR, MVS, IS, HW, HC, JCR, PF, NH, FT and LJ. KK, MBG, MK, AN, SP, WR, MVS, IS, HW, HC, JCR, PF, $\mathrm{NH}, \mathrm{FT}$ and $\mathrm{LJ}$ approved the final version of the manuscript before its submission. An AstraZeneca team reviewed the manuscript during its development and was allowed to make suggestions. However, the final content was determined by KK, MBG, MK, AN, SP, WR, MVS, IS, HW, HC, JCR, PF, NH, FT and LJ. KK is the guarantor of this work.

Funding The DISCOVER study programme is funded by AstraZeneca. DISCOVER is a non-interventional study programme and no drugs were supplied or funded. All statistical analyses were funded by AstraZeneca and conducted independently of the study sponsor, by the statistical group at Saint Luke's Mid America Heart Institute, Kansas City, Missouri, USA.

Competing interests KK, MBG, MK, AN, SP, WR, MVS, IS, HW and LJ are members of the DISCOVER Scientific Committee and received financial support from AstraZeneca to attend DISCOVER planning and update meetings. $\mathrm{HC}$ and PF are employees of AstraZeneca. NH is a former employee of AstraZeneca. JC- $\mathrm{R}$ is an employee of Evidera. In addition, KK has received honoraria from AstraZeneca, Boehringer Ingelheim, Eli Lilly, Janssen, Merck Sharp \& Dohme, Novartis, Novo Nordisk, Sanofi, Takeda, Servier and Pfizer, and research support from AstraZeneca, Boehringer Ingelheim, Eli Lilly, Merck Sharp \& Dohme, Novartis, Novo Nordisk, Sanofi and Pfizer, and also acknowledges support from the National Institute for Health Research Collaboration for Applied Research Collaboration-East Midlands (NIHR ARC-EM) and the National Institute of Health Research (NIHR) Leicester Biomedical Research Centre. MBG has received honoraria from Merck Serono. MK has received honoraria from Amgen, Applied Therapeutics, AstraZeneca, Bayer, Boehringer Ingelheim, Eisai, GlaxoSmithKline, Glytec Systems, Intarcia, Janssen, Merck (Diabetes), Novartis, Novo Nordisk, Sanofi and Vifor, and research support from AstraZeneca and Boehringer Ingelheim. AN has received honoraria from AstraZeneca, Eli Lilly, Medtronic and Novo Nordisk, and research support from Artsana, Dexcom, Novo Nordisk and Sanofi. WR has received research support from Novo Nordisk. MVS has received honoraria from AstraZeneca, Boehringer Ingelheim, Eli Lilly, Merck Sharpe \& Dohme, Novo Nordisk, Sanofi and Servier, and research support from Novo Nordisk and Sanofi. IS has received honoraria from Astellas Pharma, AstraZeneca, Boehringer Ingelheim, Kowa, Merck Sharp \& Dohme, Mitsubishi Tanabe Pharma, Novo Nordisk, Ono Pharmaceutical, Sanwa Kagaku Kenkyusho and Takeda Pharmaceutical, and research support from Astellas Pharma, AstraZeneca, Daiichi Sankyo, Eli Lilly, Japan Foundation for Applied Enzymology, Japan Science and Technology Agency, Kowa, Kyowa Hakko Kirin, Midori Health Management Center, Mitsubishi Tanabe Pharma, Novo Nordisk, Ono Pharmaceutical, Sanofi, Suzuken Memorial Foundation and Takeda Pharmaceutical.
HW has received honoraria from Astellas Pharma, AstraZeneca, Boehringer Ingelheim, Daiichi Sankyo, Dainippon Sumitomo Pharma, Eli Lilly, Kissei Pharma, Kowa, Kyowa Hakko Kirin, Merck Sharp \& Dohme, Mitsubishi Tanabe Pharma, Novartis, Novo Nordisk, Ono Pharmaceutical, Sanofi, Sanwa Kagaku Kenkyusho and Takeda, and research support from Abbott, Astellas Pharma, AstraZeneca, Bayer, Benefit One Health Care, Boehringer Ingelheim, Bristol-Myers Squibb, Daiichi Sankyo, Dainippon Sumitomo Pharma, Eli Lilly, Johnson \& Johnson, Kissei Pharma, Kowa, Kyowa Hakko Kirin, Merck Sharp \& Dohme, Mitsubishi Tanabe Pharma, Mochida Pharmaceutical, Nitto Boseki, Novartis, Novo Nordisk, Ono Pharmaceutical, Pfizer, Sanofi, Sanwa Kagaku Kenkyusho, Taisho Toyama Pharmaceutical, Takeda and Terumo Corp. FT is an employee of the Mid America Heart Institute and has received research support from AstraZeneca. LJ has received honoraria from AstraZeneca, Bayer, Boehringer Ingelheim, Bristol-Myers Squibb, Eli Lilly, Merck Sharp \& Dohme, Novartis, Novo Nordisk, Takeda, Sanofi and Roche, and research support from AstraZeneca, Bristol-Myers Squibb, Eli Lilly, Merck Sharp \& Dohme, Novartis, Roche and Sanofi.

Patient and public involvement Patients and/or the public were not involved in the design, or conduct, or reporting or dissemination plans of this research.

Patient consent for publication Not required.

Ethics approval The study protocols were approved by the relevant clinical research ethics committees in each country and institutional review boards at each site, and complied with the Declaration of Helsinki, the International Conference on Harmonisation Guideline for Good Clinical Practice and the local regulations for clinical research.

Provenance and peer review Not commissioned; externally peer reviewed.

Data availability statement Data may be obtained from a third party and are not publicly available. Data underlying the findings described in this manuscript may be obtained in accordance with AstraZeneca's data sharing policy described at https:// astrazenecagrouptrials.pharmacm.com/ST/Submission/Disclosure.

Open access This is an open access article distributed in accordance with the Creative Commons Attribution Non Commercial (CC BY-NC 4.0) license, which permits others to distribute, remix, adapt, build upon this work non-commercially, and license their derivative works on different terms, provided the original work is properly cited, appropriate credit is given, any changes made indicated, and the use is non-commercial. See: http://creativecommons.org/licenses/by-nc/4.0/.

\section{ORCID iD}

Kamlesh Khunti http://orcid.org/0000-0003-2343-7099

\section{REFERENCES}

1 International Diabetes Federation. IDF diabetes atlas. Available: http://www.diabetesatlas.org/ [Accessed 17 Jan 2020].

2 Garber AJ, Abrahamson MJ, Barzilay JI, et al. Consensus statement by the American Association of Clinical Endocrinologists and American College of Endocrinology on the comprehensive type 2 diabetes management algorithm - 2018 executive summary. Endocr Pract 2018;24:91-120.

3 International Diabetes Federation. Global guideline for type 2 diabetes. Available: https://www.idf.org/e-library/guidelines/79global-guideline-for-type-2-diabetes.html [Accessed 17 Jan 2020].

4 Davies MJ, D'Alessio DA, Fradkin J, et al. Management of hyperglycemia in type 2 diabetes, 2018. A consensus report by the American Diabetes Association (ADA) and the European Association for the Study of Diabetes (EASD). Diabetes Care 2018;41:2669-701.

5 Qaseem A, Barry MJ, Humphrey LL, et al. Oral pharmacologic treatment of type 2 diabetes mellitus: a clinical practice guideline update from the American College of Physicians. Ann Intern Med 2017;166:279-90.

6 American Diabetes Association. Standards of medical care in diabetes. Diabetes Care 2017;40:S1-129.

7 Ji L, Bonnet F, Charbonnel B, et al. Towards an improved global understanding of treatment and outcomes in people with type 2 diabetes: rationale and methods of the DISCOVER observational study program. J Diabetes Complications 2017;31:1188-96.

8 World Health Organization. World health statistics. Available: https:// www.who.int/gho/publications/world_health_statistics/en/ [Accessed 17 Jan 2020].

9 Larsen K, Merlo J. Appropriate assessment of neighborhood effects on individual health: integrating random and fixed effects in multilevel logistic regression. Am J Epidemiol 2005;161:81-8. 
10 Larsen K, Petersen JH, Budtz-Jørgensen E, et al. Interpreting parameters in the logistic regression model with random effects. Biometrics 2000;56:909-14.

11 Chinese Diabetes Society. Chinese guidelines for type 2 diabetes prevention (2013). Chin J Diabetes 2014;22:2-42.

12 Bristol-Myers Squibb Company. GLUCOPHAGE (metformin hydrochloride) [package insert]. Princeton, NJ, USA: Bristol-Myers Squibb Company, 2017.

13 Inzucchi SE, Bergenstal RM, Buse JB, et al. Management of hyperglycaemia in type 2 diabetes, 2015: a patient-centred approach. update to a position statement of the American Diabetes Association and the European Association for the Study of Diabetes. Diabetologia 2015;58:429-42.

14 Lipska KJ, Bailey CJ, Inzucchi SE. Use of metformin in the setting of mild-to-moderate renal insufficiency. Diabetes Care 2011;34:1431-7.

15 Hung AM, Roumie CL, Greevy RA, et al. Comparative effectiveness of second-line agents for the treatment of diabetes type 2 in preventing kidney function decline. Clin J Am Soc Nephrol 2016;11:2177-85.
16 Dissanayake AM, Wheldon MC, Ahmed J, et al. Extending metformin use in diabetic kidney disease: a pharmacokinetic study in stage 4 diabetic nephropathy. Kidney Int Rep 2017;2:705-12.

17 Overbeek JA, Heintjes EM, Prieto-Alhambra D, et al. Type 2 diabetes mellitus treatment patterns across Europe: a population-based multidatabase study. Clin Ther 2017;39:759-70.

18 Chow CK, Ramasundarahettige C, Hu W, et al. Availability and affordability of essential medicines for diabetes across high-income, middle-income, and low-income countries: a prospective epidemiological study. Lancet Diabetes Endocrinol 2018;6:798-808.

19 Bradley JN, Edwards KL, Gunter JT, et al. Provider decisions and patient outcomes after premature metformin discontinuation. Diabetes Spectr 2017;30:17-22.

20 Rathmann W, Medina J, Kosiborod M. The DISCOVER study: diversity of sites physicians and patients. 34th International Conference on Pharmacoepidemiology \& Therapeutic Risk Management, 2018. 